\title{
Clinical efficacy of meniscus plasty under arthroscopy in middle-aged and elderly patients with meniscus injury
}

\author{
YUHUI SHI ${ }^{*}$, ZHIGANG TIAN* ${ }^{*}$ LIFAN ZHU, JINCAI ZENG, RONG LIU and JIANXIN ZHOU \\ Department of Orthopedics, The First People's Hospital of Wujiang District Suzhou, Suzhou, Jiangsu 215200, P.R. China
}

Received May 7, 2018; Accepted July 23, 2018

DOI: $10.3892 /$ etm.2018.6519

\begin{abstract}
Clinical efficacy of meniscal plasty and total meniscectomy under arthroscopy in treating middle-aged and elderly patients with meniscus injury were explored. One hundred and fifteen elderly patients with meniscal injuries treated in the First People's Hospital of Wujiang District Suzhou from May 2012 to October 2014 were retrospectively analyzed. They were randomly divided into meniscal plasty group (experimental group) and total meniscectomy group (control group). Length of stay, operation time and VAS between the two groups were compared. Knee function was evaluated before the surgery and at the final follow-up using IKDC 2000 and Lysholm score. The differences of postoperative clinical efficacy and complications in both groups were observed. Postoperative IKDC 2000 and Lysholm score in the experimental group were superior to those of control group $(\mathrm{P}<0.001)$. There was no significant difference in the relieving effect of knee pain between the two types of procedures $(\mathrm{P}>0.05)$. Shorter operation time was found in control than that of experimental group. We did not observe remarkable differences in length of stay and postoperative complication rate between the two groups $(\mathrm{P}>0.05)$. Both meniscal plasty and total meniscectomy under arthroscopy can effectively relieve knee pain in a short time. Meniscal plasty could remarkably alleviate the progression of knee osteoarthritis, maintain knee function and improve daily life of affected population. We considered that meniscal plasty should be served as the preferred approach in treating middle-aged and elderly patients with meniscus injury.
\end{abstract}

Correspondence to: Dr Jianxin Zhou, Department of Orthopedics, The First People's Hospital of Wujiang District Suzhou, 169 Park Road, Songling, Wujiang, Suzhou, Jiangsu 215200, P.R. China E-mail: myiys237@163.com

${ }^{*}$ Contributed equally

Key words: arthroscopy, meniscal plasty, total meniscectomy, knee function

\section{Introduction}

Meniscus injury is one of the most common injuries in the knee joint. In general, men are more frequently affected than women. Meniscus injury is manifested as joint swelling, quadriceps muscle atrophy, localized pain, and tenderness. Among them, the presence of localized tenderness in the internal and lateral space are the most frequent symptoms (1). Arthroscopy is a minimally invasive surgical method, which has been clinically applied for almost 50 years (2). In recent years, arthroscopy has become the most applied surgical procedure for treating meniscal injury.

The current concept of meniscus treatment considers that the normal tissue of the meniscus should be retained to the greatest extent, and the lesioned area should be surgically repaired or removed (3). Arthroscopic meniscus surgery mainly includes total meniscectomy, meniscus plasty and perforation closure, of which total meniscectomy is effective in the early remission of clinical symptoms. However, total meniscectomy under arthroscopy can damage the function of the knee joint in the clinical application, leading to increased risk of degenerative knee disease. More seriously, elevated tension required for articular cartilage after total meniscectomy would easily progress into articular cartilage degeneration or osteoarthritis (4).

For the elderly patients with meniscus injury, some researchers recommend total meniscectomy since the dehydrated and degenerative meniscus has already lost its function (5). Other researchers believe that the meniscus injury in the majority of patients is relatively slight and meniscus still retains a certain function. Total meniscectomy would accelerate osteoarthritis process. They prefer meniscal plasty to maximally retain the physiological function and alleviate joint degeneration (6). Our study enrolled 115 patients with meniscus injury treated in the First People's Hospital of Wujiang District Suzhou (Suzhou, China). We explored the pros and cons of meniscal plasty and total meniscectomy, so as to provide new directions in treating middle-aged meniscus patients.

\section{Patients and methods}

Basic characteristics. One hundred and fifteen middle-aged and elderly patients with meniscal injury treated from May 2012 to October 2014 were enrolled. These patients all received complete follow-up. Among them, 68 cases were male patients and 47 were females, with the average age of $58.31 \pm 6.62$ years 
(50-67 years). According to the type of meniscus injury, there were 12 cases of traumatic meniscus injury and 103 cases of non-traumatic meniscus injury. Ninety eight patients experienced unilateral meniscus injury and 17 experienced bilateral meniscus injuries. Ultimately, there were 70 patients (80 cases of knee joint) who received meniscus plasty under arthroscopy and 45 patients (52 cases of knee joint) received total meniscectomy under arthroscopy. All patients underwent preoperative knee X-ray and MRI examination. No significant moderate-to-severe osteoarthritis (7), and ligament injury were observed. This study was approved by the Ethics Committee of The First People's Hospital of Wujiang District Suzhou. Signed informed consents were obtained from all participants before the study.

Surgical procedure. Subjects received epidural anesthesia or general anesthesia and were in the supine position. After routine disinfection treatment, a $0.5 \mathrm{~cm}$ incision was cut on the lateral patellar ligament and arthroscope was set. Saline was injected into the joint cavity and we ultimately determined the surgical procedure based on the location, type and injury degree.

Meniscus plasty under arthroscopy: Meniscus tissue was kept approximately 5-6 $\mathrm{mm}$ in length. Meniscus tissue was retained as much as possible. The remaining meniscus was repaired using the radiofrequency knife to keep it intact (8).

Total meniscectomy under arthroscopy: The fragment was morselized using basket forceps or a large tear was removed en bloc. The anterior oblique tear was cut with arthroscopic scissors and the fragment was removed using a grasper. The anterior and posterior edges were trimmed of the meniscus to prevent stress risers in the remaining meniscal rim.

Postoperative rehabilitation: Patients were encouraged to exercise toes immediately after the surgery. Knee flexion was gradually practiced one week after the surgery. Weight-bearing exercise was encouraged 2-4 weeks later. After 12 weeks, daily activities could be restored and patients were allowed regular physical activity 6 months later.

Observation indicators. Length of stay, operation time and visual analogue score (VAS) between the two groups were compared. Knee function was evaluated before the surgery and at the last follow-up using international knee documentation committee subjective form (IKDC 2000) and Lysholm score. The differences of postoperative clinical efficacy and improvement of knee function scores before and after surgery in both groups were observed.

Statistical analysis. SPSS21.0 (Statistical Product and Service Solutions) statistical software package (IBM Corp., Armonk, NY, USA) was used for data analysis. Data were expressed as mean \pm SEM. The t-test was used to analyze the difference between two groups. The one-way ANOVA was used to analyze the data among different groups, followed by post-hoc LSD or SNK analysis. $\mathrm{P}<0.05$ was considered to indicate a statistically significant difference.

\section{Results}

Basic characteristics of subjects. One hundred and fifteen middle-aged and elderly patients with meniscal injury
Table I. General data of the two groups.

\begin{tabular}{|c|c|c|c|c|}
\hline \multirow[b]{2}{*}{ Items } & \multirow[b]{2}{*}{ No. } & \multicolumn{2}{|c|}{ Groups } & \multirow[b]{2}{*}{ P-value } \\
\hline & & Experimental & Control & \\
\hline \multicolumn{5}{|l|}{ Sex } \\
\hline Male & 68 & 43 & 25 & \multirow[t]{2}{*}{0.5317} \\
\hline Female & 47 & 27 & 20 & \\
\hline \multicolumn{5}{|l|}{ Disease date } \\
\hline$<2$ months & 49 & 30 & 19 & \multirow[t]{2}{*}{0.9464} \\
\hline$\geq 2$ months & 66 & 40 & 26 & \\
\hline \multicolumn{5}{|l|}{ Cause of disease } \\
\hline Traumatic & 12 & 5 & 7 & \multirow[t]{2}{*}{0.1498} \\
\hline Non-traumatic & 103 & 65 & 38 & \\
\hline \multicolumn{5}{|l|}{ Injured knee } \\
\hline Left & 51 & 32 & 19 & \multirow[t]{2}{*}{0.6397} \\
\hline Right & 64 & 38 & 27 & \\
\hline \multicolumn{5}{|l|}{ Injured part } \\
\hline Anterior horn & 23 & 15 & 8 & \multirow[t]{3}{*}{0.793} \\
\hline Somatic part & 62 & 36 & 26 & \\
\hline Posterior horn & 30 & 19 & 11 & \\
\hline \multicolumn{5}{|l|}{ BMI } \\
\hline$<30 \mathrm{~kg} / \mathrm{m}^{2}$ & 88 & 51 & 37 & \multirow[t]{2}{*}{0.2475} \\
\hline$\geq 30 \mathrm{~kg} / \mathrm{m}^{2}$ & 27 & 19 & 8 & \\
\hline \multicolumn{5}{|c|}{ No. of comorbidities } \\
\hline $0-1$ & 94 & 59 & 35 & \multirow[t]{2}{*}{0.3779} \\
\hline 2 & 21 & 11 & 10 & \\
\hline \multicolumn{5}{|c|}{$\begin{array}{l}\text { Kellgren-Lawrence } \\
\text { grade }\end{array}$} \\
\hline 0 & 13 & 8 & 5 & \multirow[t]{3}{*}{0.6788} \\
\hline 1 & 88 & 52 & 36 & \\
\hline 2 & 14 & 10 & 4 & \\
\hline \multicolumn{5}{|l|}{ Knee flexion } \\
\hline$<120^{\circ}$ & 11 & 6 & 5 & \multirow[t]{2}{*}{0.6513} \\
\hline$\geq 120^{\circ}$ & 104 & 64 & 40 & \\
\hline \multicolumn{5}{|l|}{ Swelling } \\
\hline No & 27 & 18 & 9 & \multirow[t]{2}{*}{0.4804} \\
\hline Yes & 88 & 52 & 36 & \\
\hline
\end{tabular}

treated in the hospital from May 2012 to October 2014 were enrolled. The average postoperative follow-up of these patients was 2.6 \pm 0.3 years (2.3-3 years). There were 70 cases in experimental group with the average age of $57.26 \pm 7.82$ years (50-65 years) and 45 cases in control group with the average age of $59.43 \pm 6.33$ years (50-67 years). No significant differences were found in sex, age and meniscus injury site between the two groups $(\mathrm{P}>0.05$; Table I).

Comparison of operation time, length of stay and recovery time. We did not observe significant differences in length of stay and recovery time between the two groups $(\mathrm{P}>0.05)$. However, shorter operation time was found in control group 

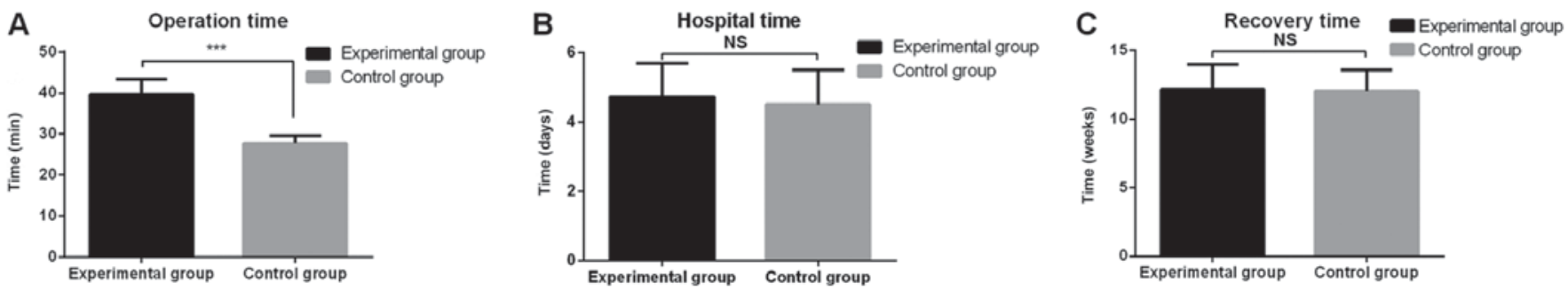

Figure 1. Comparison of operation time, length of stay and recovery time. (A) Operation time was shorter in control group than that of experimental group. (B) No significant difference in length of stay was found between the two groups. (C) No significant difference in recovery time was found between the two groups. ***** $\mathrm{P}<0.001$.

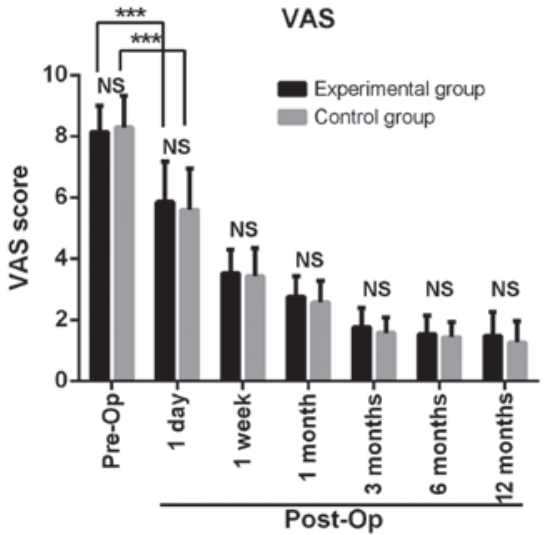

Figure 2. Comparison of VAS before, and after the surgery and in the follow-up period. Postoperative VAS in both groups was decreased compared to the preoperative ones. No significant difference in postoperative VAS was found between the two groups. VAS, visual analogue score. ${ }^{* * *} \mathrm{P}<0.001$.

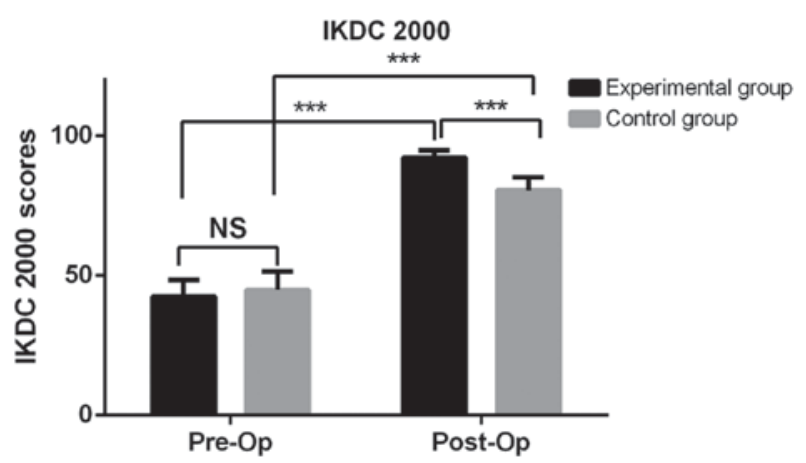

Figure 3. Comparison of IKDC 2000 before surgery and at the final follow-up. No significant difference in preoperative IKDC 2000 was found between the two groups. IKDC 2000 was significantly elevated after the surgery and higher IKDC 2000 was found in the experimental group than that of control group. ${ }^{* * *} \mathrm{P}<0.001$

than that of experimental group, indicating smaller surgical risk of total meniscectomy $(\mathrm{P}<0.05$; Fig. 1$)$.

Comparison of therapeutic efficacy. Postoperative VAS in both groups was decreased compared to the preoperative ones, which were decreased from 8.14 to 5.85 and 8.28 to 5.59 in experimental and control group, respectively $(\mathrm{P}<0.001)$. However, no significant difference in relieving postoperative pain between the two groups, indicating that meniscus plasty and total meniscectomy have similar effect on improving knee pain ( $\mathrm{P}>0.05$; Fig. 2).

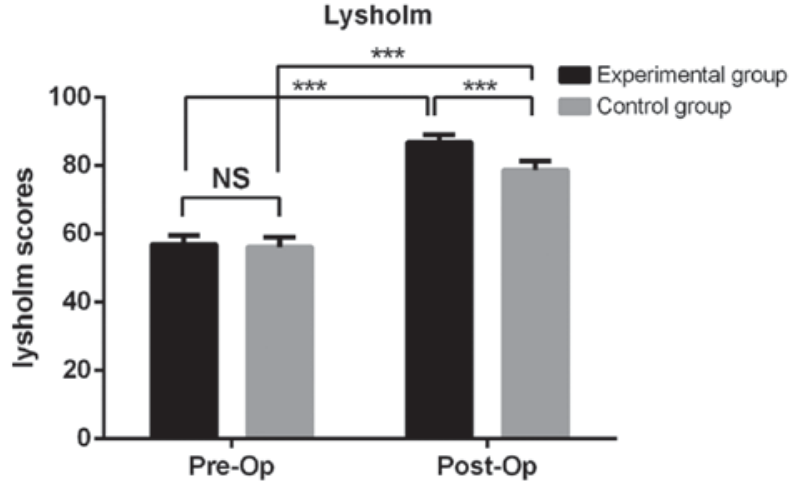

Figure 4. Comparison of Lysholm score before surgery and at the final follow-up. No significant difference in preoperative Lysholm score was found between the two groups. Lysholm score was significantly improved after surgery and higher Lysholm score was found in experimental group than that of control group. ${ }^{* * *} \mathrm{P}<0.001$.

Table II. Complications of the two groups.

\begin{tabular}{lrcrr}
\hline & & \multicolumn{3}{c}{ Groups } \\
\cline { 3 - 4 } Items & No. & Experimental & Control & P-value \\
\hline $\begin{array}{l}\text { Deep venous } \\
\text { thrombosis }\end{array}$ & 3 & 1 & 2 & 0.9051 \\
$\begin{array}{l}\text { Swelling } \\
\text { Pain }\end{array}$ & 18 & 8 & 10 & \\
\hline
\end{tabular}

IKDC 2000 at the final follow-up were decreased from 92.22 to 42.42 and 80.53 to 44.89 in experimental group and control group, respectively $(\mathrm{P}<0.001$; Fig. 3$)$. The improvement of IKDC 2000 was better in experimental than that of control group.

Postoperative Lysholm were elevated from 56.87 to 86.91 and 56.09 to 78.73 in experimental and control group, respectively $(\mathrm{P}<0.001$; Fig. 4). Significant difference was found in Lysholm at the follow-up period between the two groups, suggesting that the improvement of knee function is better in experiment group.

Comparison of postoperative complications. No infection occurred during the follow-up period in the two groups. There were some patients experiencing deep venous thrombosis, joint pain and swelling in the lower extremities within three months 
after the operation. The above symptoms gradually improved and the thrombus gradually disappeared after corresponding treatment. No significant difference in postoperative complication was found between the two groups ( $\mathrm{P}>0.05$; Table II).

\section{Discussion}

Meniscus is the fibrocartilage between the femoral condyle and the tibial plateau. Meniscus possesses important functions, such as load transition, shock absorption, joint stabilization and knee activity cooperation. Mechanical stability of knee joint would be damaged if meniscus injury or meniscus tear occurs, leading to early onset of osteoarthritis (9). Therefore, meniscal injury should be repaired as much as possible. The advantages of arthroscopic surgery for meniscus injury mainly include small incision, slight trauma and quick recovery of daily activity. Besides, arthroscopy could examine complex injuries related to cartilage and ligament injuries. Arthroscopic surgery has replaced open surgery as the best treatment for meniscal injury (10).

The vascularized area of meniscus can be classified into the red-red zone (vascular portion that is located at 1-3 mm away from meniscus synovium), red-white zone (blood supply is from the end branch of capillaries in the red-red zone, which is located 3-5 mm inside of red-red zone), and white-white zone (non-vascular portion that is located inside the red-white zone) according to the degree of vascularity. The capillary plexus gradually decreases with age. There are many great vessels in the meniscus before 14 years. However, $75 \%$ of great vessels gradually disappear from the center of the meniscus after 14 years. The meniscus vascular area is only $10-30 \%$ of the periphery of the meniscus in adulthood $(11,12)$. This special anatomy of meniscus makes it difficult for repair of middle-aged patients with meniscal tear. However, meniscus still retains its value since peripheral vascular supply is functional. In the present study, meniscus tissue was kept approximately $5-6 \mathrm{~mm}$ in length. We tried to retain the meniscus function so as to reduce the sequelae after meniscectomy.

Pathological changes of meniscus lesions in middle-aged and elderly patients are remarkably different from those of young adults. The majority of meniscal injuries in young adults are caused by direct or indirect violence. Meniscus injuries in middle-aged and elderly patients are commonly related to meniscus degeneration. Loss of moist, thinner layer and decreased elasticity all lead to adhesion and poor mobility of meniscus. Moreover, decreased elastic strain and buffering effect further exaggerate meniscus tear or wear (13). Patients with meniscus injury are manifested as knee pain, pressure pain at the joint line, limited range of knee flexure and knee swelling. In some severe cases, locked joint and joint dysfunction are observed (14). In our study, only $12 / 115$ patients were traumatic meniscus injury cases. Other patients had no obvious trauma history and their meniscus injuries resulted from chronic strain and degenerative causes, which was consistent with other reports (15). Among them, $104(90.43 \%)$ cases presented limited knee extension function and $88(76.52 \%)$ presented swelling knee joint. The diagnosis of meniscus injury could be confirmed with other vital signs and MRI examination (16).
Untimely treatment of meniscus injury would lead to aggravated meniscus tear during repeated flexion and extension of the knee joint. The torn meniscus also result in joint cartilage damage to the femur and tibia due to the compressive force, eventually leading to severe secondary articular cartilage injury $(17,18)$. Higuchi et al (19) performed a 12.2-year follow-up and found that the amount of resected meniscus is one of the decisive factors affecting the therapeutic effect. After the total meniscectomy, axial alignment of the lower extremity was remarkably changed, which was closely related to the resected area of meniscus. Baratz et al (20) found that the contact area of the femoral condyle articular surface is reduced by $75 \%$, and the articular surface contact stress is increased to $235 \%$ after total meniscectomy. The role of the meniscus in receiving and conducting the load disappears, resulting in a significant change in the axial alignment of the lower extremity. However, the contact area is only reduced by $10 \%$, and the contact stress is increased by $65 \%$ after partial meniscectomy $(20,21)$. Alterations of axial alignment of the lower extremity result in damage to articular cartilage in the non-weight-bearing region of the femur and tibia, and eventually lead to osteoarthritis. The occurrence of osteoarthritis gradually leads to swelling, pain and limited functional activities of the knee joint, which further affects the daily life of affected population. In the present study, IKDC 2000 and Lysholm scores were better in the experimental group than those of the control group at the final follow-up. Our data indicated that the partial meniscus retained in the meniscus plasty can effectively alleviate the knee joint degeneration and progress of arthritis to maintain knee function.

Arthroscopy is an effective approach to treat knee joint diseases. It has the advantages of high efficiency and minimal invasiveness. Although the proportion of postoperative complications of knee arthroscopic surgery is approximately $2 \%$, it is clinically significant due to the dramatic increase in the number of knee arthroscopic surgeries. There are also many cases of postoperative complications of knee arthroscopic surgery (22). Small (23) researched 10,262 cases of arthroscopic surgery (of which, 8,741 cases underwent knee arthroscopy). The incidence of postoperative complications was $1.68 \%$. Among them, joint hemostasis, infection and thromboembolism occurred most frequently, which accounted for $60.1,12.1$ and $6.9 \%$, respectively. In our study, there were $3(2.6 \%)$ cases of deep vein thrombosis, 8 (6.9\%) of knee pain and 18 (15.6\%) of knee swelling. No significant adverse effect was found after anticoagulant local physiotherapy was given.

Arthroscopic surgery, as a minimally invasive surgery, and requires the operator to strictly control surgical indications. Proficient in knee arthroscopy and careful procedures can minimize the occurrence of postoperative complications and comprehensively reflect the minimally invasive value of arthroscopic knee surgery. Meniscus plasty and total meniscectomy under arthroscopy can effectively improve knee joint pain symptoms in a short period of time. In particular, meniscus plasty remarkably alleviates the occurrence of knee osteoarthritis, maintains the knee joint function, and improves the daily activity of affected patients. Meniscus plasty should be the preferred treatment for elderly patients with meniscus injury who do not have significant moderate and severe osteoarthritis. Furthermore, studies with large sample and long-term follow-up 
data are still needed for the further exploration of the long-term knee function changes in patients with meniscal injury.

In conclusion, both meniscal plasty and total meniscectomy under arthroscopy can effectively relieve knee pain in a short time. Meniscal plasty could remarkably alleviate the progression of knee osteoarthritis, maintain knee function and improve daily life of affected population. We consider that meniscal plasty should be the preferred approach in treating middle-aged and elderly patients with meniscus injury.

\section{Acknowledgements}

Not applicable.

\section{Funding}

No funding was received.

\section{Availability of data and materials}

All data generated or analyzed during this study are included in this published article.

\section{Authors' contributions}

YS, ZT and JZh designed the study and performed the experiments. LZ and JZe collected the data. ZT and RL analyzed the data. YS and ZT prepared the manuscript. All authors read and approved the final manuscript.

\section{Ethics approval and consent to participate}

This study was approved by the Ethics Committee of The First People's Hospital of Wujiang District Suzhou (Suzhou, China). Signed informed consents were obtained from the patients or the guardians.

\section{Patient consent for publication}

Not applicable.

\section{Competing interests}

The authors declare that they have no competing interests.

\section{References}

1. Rohren EM, Kosarek FJ and Helms CA: Discoid lateral meniscus and the frequency of meniscal tears. Skeletal Radiol 30: 316-320, 2001.

2. Johnson MJ, Lucas GL, Dusek JK and Henning CE: Isolated arthroscopic meniscal repair: A long-term outcome study (more than 10 years). Am J Sports Med 27: 44-49, 1999.

3. Kollias SL and Fox JM: Meniscal repair. Where do we go from here? Clin Sports Med 15: 621-630, 1996.

4. Rangger C, Klestil T, Gloetzer W, Kemmler G and Benedetto KP: Osteoarthritis after arthroscopic partial meniscectomy. Am J Sports Med 23: 240-244, 1995.

5. Bin SI, Lee SH, Kim CW, Kim TH and Lee DH: Results of arthroscopic medial meniscectomy in patients with grade IV osteoarthritis of the medial compartment. Arthroscopy 24: 264-268, 2008

6. Englund M: Meniscal tear - a feature of osteoarthritis. Acta Orthop Scand Suppl 75: 1-45, 2004.
7. Hochberg MC, Altman RD, Brandt KD, Clark BM, Dieppe PA, Griffin MR, Moskowitz RW and Schnitzer TJ; American College of Rheumatology: Guidelines for the medical management of osteoarthritis. Part II. Osteoarthritis of the knee. Arthritis Rheum 38: 1541-1546, 1995.

8. Krych AJ, Bert JM and Levy BA: Treatment of OA of the knee in the middle-aged athlete: The role of arthroscopy. Sports Med Arthrosc Rev 21: 23-30, 2013.

9. McDermott ID and Amis AA: The consequences of meniscectomy. J Bone Joint Surg Br 88: 1549-1556, 2006.

10. Nepple JJ, Dunn WR and Wright RW: Meniscal repair outcomes at greater than five years: A systematic literature review and meta-analysis. J Bone Joint Surg Am 94: 2222-2227, 2012.

11. Arnoczky SP and Warren RF: Microvasculature of the human meniscus. Am J Sports Med 10: 90-95, 1982.

12. Arnoczky SP and Warren RF: The microvasculature of the meniscus and its response to injury. An experimental study in the dog. Am J Sports Med 11: 131-141, 1983.

13. Maffulli N, Longo UG, Campi S and Denaro V: Meniscal tears. Open Access J Sports Med 1: 45-54, 2010.

14. Levy IM, Torzilli PA and Warren RF: The effect of medial meniscectomy on anterior-posterior motion of the knee. J Bone Joint Surg Am 64: 883-888, 1982.

15. Englund M, Roemer FW, Hayashi D, Crema MD and Guermazi A: Meniscus pathology, osteoarthritis and the treatment controversy. Nat Rev Rheumatol 8: 412-419, 2012.

16. Bhattacharyya T, Gale D, Dewire P, Totterman S, Gale ME, McLaughlin S, Einhorn TA and Felson DT: The clinical importance of meniscal tears demonstrated by magnetic resonance imaging in osteoarthritis of the knee. J Bone Joint Surg Am 85-A: 4-9, 2003.

17. Krause WR, Pope MH, Johnson RJ and Wilder DG: Mechanical changes in the knee after meniscectomy. J Bone Joint Surg Am 58: 599-604, 1976.

18. McCarty EC, Marx RG and DeHaven KE: Meniscus repair: Considerations in treatment and update of clinical results. Clin Orthop Relat Res 402: 122-134, 2002.

19. Higuchi H, Kimura M, Shirakura K, Terauchi M and Takagishi K: Factors affecting long-term results after arthroscopic partial meniscectomy. Clin Orthop Relat Res 377: 161-168, 2000.

20. Baratz ME, Fu FH and Mengato R: Meniscal tears: The effect of meniscectomy and of repair on intraarticular contact areas and stress in the human knee. A preliminary report. Am J Sports Med 14: 270-275, 1986.

21. Ahmed AM and Burke DL: In-vitro measurement of static pressure distribution in synovial joints - Part I: Tibial surface of the knee. J Biomech Eng 105: 216-225, 1983.

22. Pierzchała A, Kusz D and Widuchowski J: Complication of arthroscopy of the knee. Wiad Lek 56: 460-467, 2003 (In Polish).

23. Small NC: Complications in arthroscopic surgery performed by experienced arthroscopists. Arthroscopy 4: 215-221, 1988.

This work is licensed under a Creative Commons Attribution-NonCommercial-NoDerivatives 4.0 International (CC BY-NC-ND 4.0) License. 